\title{
THE EXPRESSION ANALYSIS OF PERI GENE IN FUGU, TAKIFUGU RUBRIPES ADMINISTERED WITH IMMUNOSTIMULANTS
}

\author{
Goshi Nishi $^{1}$, Jun-ichi Hikima ${ }^{2}$, Masahiro Sakai ${ }^{2}$, Tomoya Kono ${ }^{2 \S}$ \\ ${ }^{1}$ Course of Biochemistry and Applied Biosciences, Graduate School of Agriculture, University of \\ Miyazaki, Miyazaki 889-2192, Japan \\ ${ }^{2}$ Department of Biochemistry and Applied Biosciences, Faculty of Agriculture, University of \\ Miyazaki, Miyazaki 889-2192, Japan
}

\begin{abstract}
Circadian rhythms are physical, mental and behavioral changes that follow a roughly 24-hour cycle, responding primarily to light and darkness in an organism's environment. These changes occur in most living organisms, including animals, plants and many microbes. Circadian rhythm oscillations are the daily changes in physiology and are due to involvement of numerous genes whose expression peaks and declines approximately $12 \mathrm{hrs}$ apart to undergo a full cycle within $24 \mathrm{hrs}$. These circadian rhythm controlling genes are called as clock genes that include Bmal, Clock, Period (per) and Cry genes in mammals. In recent years, it has been known that clock genes regulate various physiological activities including immune response. But there is little or no information on these genes and their functions in fish. Therefore, cloning of the Japanese pufferfish, Takifugu rubripes perl gene was conducted, and expression pattern of the gene in light and dark periods with/without immune stimulation by LPS or polyI:C was analyzed in this study. Results revealed that the per 1 gene was composed of 4215 bp nucleotides that encode a 1404-amino acid residue. A rhythmic change of perl gene expression was observed during light and dark periods. A phase change (gap) of perl gene expression was confirmed in the pufferfish stimulated with LPS. But a gap of perl gene expression was not confirmed in polyI:C stimulated fish. It is suggested that perl gene participates in immune regulation at the time of the bacterial infection but not during viral infection because a phase change was seen only in LPS stimulated fish. In conclusion, fish perl gene may have regulatory function in immune response similar to that of mammalian species.
\end{abstract}

\section{KEYWORDS}

Period1 (per1) gene, circadian rhythm, LPS stimulation, rhythmic change, phase change (gap)

§Corresponding author. Tel/Fax: +81-985-587866

E-mail address: tkono@cc.miyazaki-u.ac.jp 